\title{
A Common Mechanism for Illusory and Occluded Object Completion
}

\author{
Philip J. Kellman and Carol Yin \\ University of California, Los Angeles
}

\author{
Thomas F. Shipley \\ Temple University
}

\begin{abstract}
New phenomena and results are reported that implicate a common contour interpolation mechanism in illusory and occluded (modal and amodal) object completion. In 3 experiments, a speeded classification task was used to study novel quasimodal displays in which occluded and illusory contours join. Results showed the same advantages in speed and accuracy over control displays for quasimodal, illusory, and occluded displays. The implications of quasimodal displays, along with another new display type in which contour linkages must precede determination of modal or amodal appearance, are considered. These logical considerations and empirical results suggest that amodal and modal completion depend on a common underlying mechanism that connects edges across gaps.
\end{abstract}

A number of visual phenomena share the property that boundaries and shape are perceived in locations where no local information is present. These gaps in the projected boundaries and surfaces of objects often go unnoticed because of visual completion processes that deliver representations of complete boundaries, whole objects, and continuous surfaces. Some examples are partly occluded objects, illusory objects, some cases of apparent transparency, and spontaneously splitting objects (SSOs). For the central figure seen in each example in Figure 1, the four displays are equivalent in terms of the contours and gaps given in the stimulus. These visual phenomena have often been considered to be manifestations of different processes. Michotte, Thines, and Crabbe (1964), emphasizing differences in their phenomenology, divided completion processes into two types. Objects that are completed modally have a sensory presence in the areas that lack local specification. In the illusory figure in Figure 1b, for example, one can note the apparent lightness of the completed surface or answer a question about whether that surface has a small blue spot on it. (It does not.) In cases of amodal completion, one perceives or registers unspecified parts of objects, but these do not have a sensory presence. In Figure 1a, the black parts are perceived as connecting behind the occluder, but this connection is hidden. One would not be able to answer the

Philip J. Kellman and Carol Yin, Department of Psychology, University of California, Los Angeles; Thomas F. Shipley, Department of Psychology, Temple University. Carol Yin is now at Computation and Neural Systems, Division of Biology, Califomia Institute of Technology.

Portions of this research were presented at the annual meeting of the Association for Research in Vision and Ophthalmology, May 1995. We gratefully acknowledge support from National Science Foundation Research Grants SBR 9496112 and SBR 9396309.

We thank Barton Anderson, Joseph Lappin, Robert Shapley, and an anonymous reviewer for helpful comments and suggestions, and we thank Paras Doshi and Shannon White for assistance in data collection.

Correspondence concerning this article should be addressed to Philip J. Kellman, Department of Psychology, University of California, 405 Hilgard Avenue, Los Angeles, California 900241563. Electronic mail may be sent to Kellman@ cognet.ucla.edu. question about the presence of a blue spot in the middle of the partly occluded object in Figure 1a. Another way of making the distinction is that a modally completed edge or surface is the nearest edge or surface to the observer in a particular visual direction, whereas an amodally completed edge or surface is not (i.e., it is located behind some nearer surface).

Differences in their phenomenal appearances have led to different explanations for these phenomena. Illusory contours have usually been explained in terms of basic sensory mechanisms either at the retina (Brigner \& Gallagher, 1974; Jory \& Day, 1979) or in early cortical visual areas (von der Heydt, Peterhans, \& Baumgartner, 1984). Completion of partly occluded contours and objects has sometimes been considered to be more cognitive in origin, perhaps not explainable by truly sensory or perceptual mechanisms at all (Gregory, 1977; Rock, 1987). Some theorists, however, have viewed both kinds of phenomena in terms of autonomous perceptual mechanisms (Kanizsa, 1979; Michotte et al., 1964) or cognitively oriented approaches to perception (Rock, 1987).

Several investigators have proposed that boundaries and surfaces play complementary roles in completion: Boundaries are interpolated across gaps, and surface qualities (e.g., lightness and color) fill in within completed boundaries (Grossberg \& Mingolla, 1985; Kellman \& Shipley, 1991; Yarbus, 1967; Yin, Kellman, \& Shipley, 1997). Earlier we proposed that amodal and modal boundary completion depend on a common underlying process (Kellman \& Loukides, 1987; Kellman \& Shipley, 1991; Shipley \& Kellman, 1992). Some definitions and concepts may be helpful in understanding this claim. The parts of boundaries signaled by differences in luminance, chromaticity, texture, depth, or motion we call physically specified boundaries. By interpolated boundaries we mean perceived contours that do not have local physical specification, that is, those that are created by the visual system. The claim that a common mechanism completes boundaries in the phenomenologically different cases described above we call the identity hypothesis (Shipley \& Kellman, 1992). On this hypothesis, the differences between modal and amodal phenomena have 


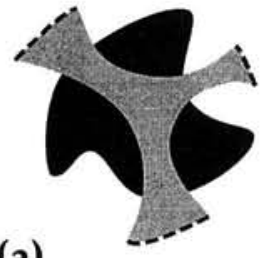

(a)

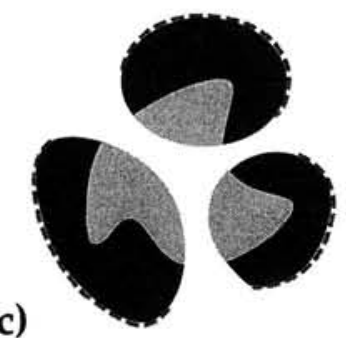

(b)

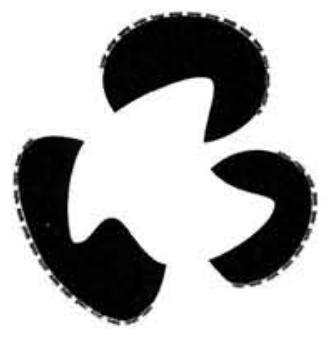

(d)

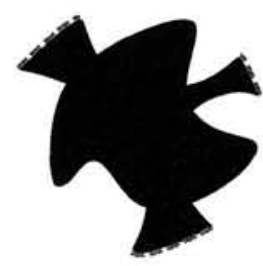

Figure 1. Equivalent object completion displays with differing appearances: (a) partly occluded object, (b) illusory object, (c) transparent illusory object, (d) spontaneously splitting object. All displays have identical contours except those shown with dotted markings.

to do with whether interpolated boundaries are in front of or behind other surfaces and not with the mechanisms that interpolate boundaries. The depth relations determining phenomenal appearance depend on depth information in the scene.

Support for a common mechanism in illusory and occluded object perception has come from three sources. One source involves arguments based on certain bistable displays, invariably involving weak or absent depth order information among visible surfaces. Figure 2 shows an example of an SSO. There are several interesting features of this display. First, it appears to segregate into two objects, despite consisting of a single, physically homogeneous region. This segregation has been explained in terms of properties of the boundary interpolation process (Kellman \& Shipley, 1991). With prolonged viewing, another property is evident. The depth ordering of the two objects switches. Sometimes the vertical object is on top (nearer the observer), and sometimes the horizontal object is on top.

If modal and amodal completion are taken to name separate visual processes, this depth reversal is a complex process indeed. The object seen on top is modally completed, whereas the other is amodally completed. When the reversal occurs, the object previously completed modally ceases to be completed that way and now becomes amodally completed. With perfect complementarity the other object undergoes the reverse change. One would need an explanation of the boundary formation process in modal completion, the parallel process in amodal completion, and some explanation of their linkage.

A simpler hypothesis is that there are not two interpolation processes here, but only one. The differing appearances of the two objects formed depend on their ordering in depth. The display in Figure 2 lacks information specifying depth order. Despite its absence (or despite the information for equal depths given by oculomotor, stereoscopic, and motion cues), the visual system appears to obey a constraint that one object or the other must appear in front. Which object appears nearer shifts across time. Depth reversals under such circumstances are characteristic of other perceptual phenomena, such as the Schrödinger staircase or reversible figureground displays. These changes of depth order in SSO displays do not alter interpolated boundaries or shapes of completed objects. The same bounded objects appear in the display, but their positions have changed.

Besides the simplicity of the identity hypothesis, two sorts of experimental evidence have been offered in its support. Using perceptual report measures, Shipley and Kellman (1992) tested a large sample of randomly generated illusory objects and corresponding partly occluded objects. For displays having the same physically specified edges, magnitude ratings of contour clarity in illusory contour displays nearly perfectly predicted ratings of perceived unity in occlusion displays.

More recently, a method based on objective performance has provided support for the identity hypothesis (Ringach \& Shapley, 1996; Shapley \& Ringach, 1994). Ringach and Shapley used displays like those shown in Figure 3. All displays were made of identical illusory contour or occluded contour inducing elements, rotated around their centers to create displays whose middles were "fat" or "thin," as shown in Figure 3a and 3b, respectively. Amount of rotation of the elements was varied. On each trial, a display was presented briefly, and participants classified it as fat or thin.

Ringach and Shapley (1996) reported that accuracy for different degrees of rotation showed the same patterns for occluded (amodally completed) and illusory (modally completed) figures. Both kinds of figures produced performance superior to that of a control group. In the control group, elements were reflected around vertical axes to disrupt figural completion. (The concavity in each three-quarter circle faced outward.) Displays could still be judged, however, as fat or thin on the basis of the same

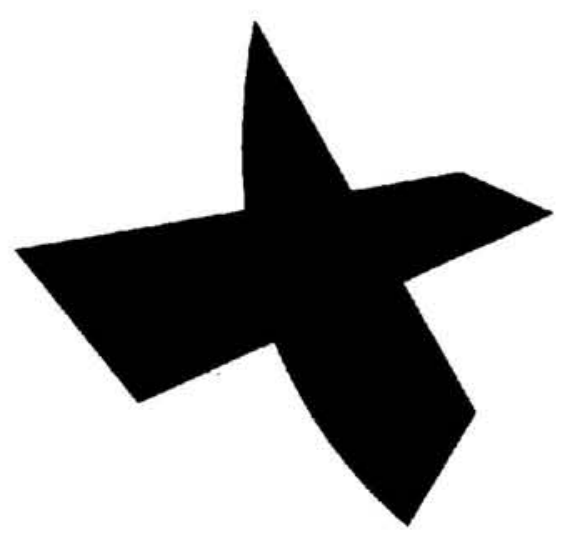

Figure 2. Bistable spontaneously splitting object display. (Redrawn from Kellman \& Shipley, 1991.) 
information about local element rotation that was available in the displays of illusory and occluded objects. Ringach and Shapley argued that the lower performance in the control group indicates that performance on this task is facilitated by global figural completion. The results in the figural completion conditions supported the notion that a single mechanism produces both kinds of contour interpolation.

There have been, however, some claims of an asymmetry in edge interpolation. For example, Anderson and Julesz (1995) reported an asymmetry in the stability of interpolation of a horizontal bar, depending on whether stereoscopic depth information places the endpoints of the horizontal bar nearer or farther than a vertical bar. In the former case, the bar may appear modally completed in front of the vertical or may take on a different ("bent") appearance. In the latter case, there is only one appearance-a straight continuation of the horizontal bar. It is not clear how readily the bent percept is seen in the less stable case. Nakayama and Shimojo $(1990,1992)$ informally presented the same display to several hundred observers and reported that "only a tiny minority" observed the horizontal bar as being bent (Nakayama \& Shimojo, 1992, p. 1358). Although the difference in stability may indicate some difference in how boundary interpolation works in the two cases, it may instead involve interactions of depth spreading along the horizontal boundary, boundary interpolation, and attentional scrutiny.

Evidence consistent with the identity hypothesis does not rule out the idea that separate but similar mechanisms produce illusory and occluded contours. Our purpose in this article is to introduce new logical and empirical considerations that place the identity hypothesis on firmer ground. Specifically, we present a new class of display in which illusory and occluded contours join. Second, we test figural completion in this new class of displays and compare it with completion performance in illusory and occluded object

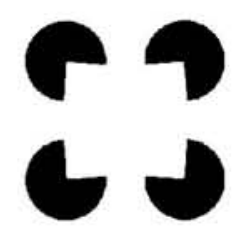

(a)

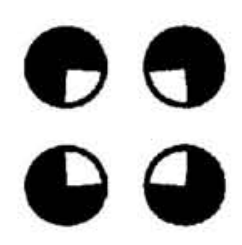

(c)

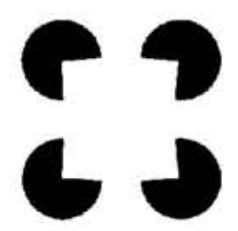

(b)

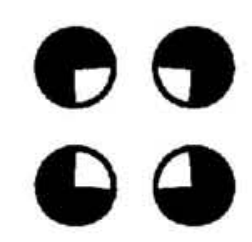

(d)
Figure 3. Stimuli used by Ringach and Shapley (1996): (a) fat illusory object, (b) thin illusory object, (c) fat partly occluded object, (d) thin partly occluded object.

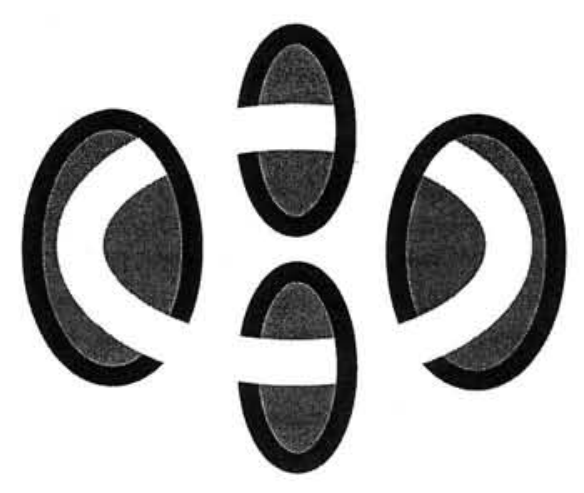

Figure 4. A quasimodal display in which modally and amodally completed contours join. The white, tubular object is completed across four gaps: In the upper right, it has partly occluded surfaces, and in the lower left, it has illusory surfaces. In the other two locations, a quasimodal connection (part occluded, part illusory) occurs.

displays. Third, we introduce another new type of display and consider some earlier phenomena to demonstrate that there are cases in which contour completion must precede determination of whether the completed boundaries and surfaces are illusory (modal) or occluded (amodal). These logical and empirical considerations implicate a common contour completion process that is neither modal nor amodal but that underlies both of these types of contour completion.

\section{Experiment 1}

Consider the display shown in Figure 4. Some of the completed contours connect a modal (illusory) portion and an amodal (occluded) portion. Logically, the existence of such connections suggests that amodal and modal completion are not separate processes. One reason is that the amodal and modal parts of the contour are able to connect. A more important reason is that this connection occurs in displays that do not fulfill the requirements for interpolation of either illusory or occluded contours. Illusory contours ordinarily begin and end with illusory contour inducing elements, and completed occluded contours begin and end at two visible contours on opposite sides of an occluder. ${ }^{1}$ On the assumption that illusory contour and occluded contour completion derive from separate mechanisms, no edge completion should occur across the upper left and lower right gaps in Figure 4 because they do not fulfill the required conditions for either mechanism to operate. Put more positively, if interpolation occurs between an illusory con-

${ }^{1}$ We distinguish between amodal completion of a boundary, which requires visible anchors at both ends of the boundary, and amodal continuation, in which a contour of a single visible part may appear to continue through a $T$ junction behind a surface to an indefinite endpoint (Kanizsa, 1979; Shimojo \& Nakayama, 1990). Our argument clearly involves completion: There are no examples in which amodal continuation (from a single visible part) alone leads to a boundary or surface emerging on the other side of an occluder. A related point is that the continuation process does not seem to occur for single illusory contour elements. 
tour inducer and an occluded contour inducer, it suggests that a single interpolation process can accept concurrently these two types of contour anchors as inputs. Thus, the completed boundaries and figures are neither strictly modal nor amodal. They are both, or neither, or perhaps quasimodal.

It remains to be shown, however, that figural completion actually occurs in quasimodal displays. Although we might claim that the reader can verify this by looking at an example, a stronger form of evidence would be to show that such quasimodal displays have similar effects on speeded performance as do other acknowledged examples of figural completion. Moreover, if a single completion mechanism governs occluded, illusory, and quasimodal displays, we would predict similar patterns of performance for the three kinds of displays.

To test these hypotheses, we used a version of the fat-thin task developed by Ringach and Shapley (1996). We used three classes of displays: illusory, occluded, and quasimodal. Four amounts of rotation were used: $2^{\circ}, 3^{\circ}, 4^{\circ}$, or $8^{\circ}$ of rotation of the corner elements around their centers. Displays are shown in Figure 5. Because completion in the quasimodal displays would lead to a surface that appears in front of two of the corner elements and behind parts of two others, we were concerned that the absence of depth differences among inducers might make the completed object appear distorted. Accordingly, we also tested displays in each class in which stereoscopic depth differences were added. Illusory contour inducing elements were made to appear farther away than the surface of the illusory figure by introducing crossed disparity for the interior edges of the inducing elements relative to the outer edges. For corner

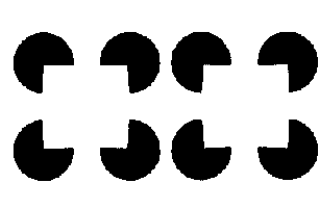

(a)

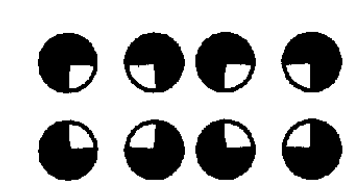

(b)

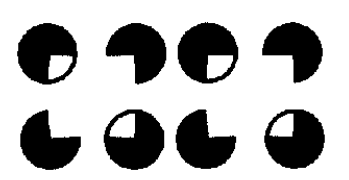

(c)

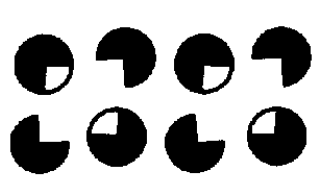

(d)

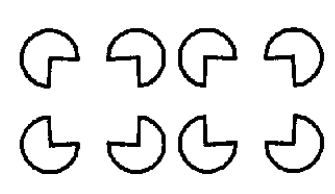

(e)
Figure 5. Stimuli used in current experiments. Stereopairs used in the depth conditions are shown. Each half illustrates the display's appearance in the no-depth conditions: (a) and (b) Illusory and occluded stimuli, similar to those used by Shapley and Ringach (1994). (c) Quasimodal display used in Experiments 1 and 2. (d) Misaligned-element control display used in Experiment 1. (e) Outline control display used in Experiment 2. elements giving rise to occluded contours, we introduced uncrossed disparity of interior edges relative to the outer edges.

A separate purpose of this experiment was to further validate the fat-thin method by using a different kind of control stimulus. The type of control display used by Ringach and Shapley (1996) had the same local elements but in different orientations from the completion displays. Concavities of the inducing elements, for example, all faced inward (toward the center) in completion displays, but half faced inward and half outward in their control displays. It is possible that these positional differences made determination of element rotation more difficult.

More comparable positioning is possible with control displays having slightly misaligned edges. On the basis of earlier research, we expected that misalignment of parallel contours should disrupt object completion. Specifically, the completion process is disrupted by small misalignments (shifts perpendicular to orientation) of collinear edges (Shipley \& Kellman, 1992). In the perceptual report (magnitude estimation) paradigm used by Shipley and Kellman, completion effects dropped rapidly with departures from collinearity and were virtually eliminated by misalignment of about $15 \mathrm{~min}$. A secondary benefit of using a misalignedelement control group in this experiment is that it allowed us to assess these earlier observations in an objective performance task, which has not previously been done.

\section{Method}

Participants. Fifteen male and female undergraduates participated in partial fulfillment of requirements for an introductory psychology course at the University of California, Los Angeles. All participants had normal or corrected-to-normal vision.

Design. The design crossed four independent variables: (a) inducing element type, with four levels consisting of illusory (modal), occluded (amodal), quasimodal, and misaligned control (using quasimodal inducers that were spatially misaligned along the $x$ and $y$ axes; (b) inducer rotation, with four levels $\left(2^{\circ}, 3^{\circ}, 4^{\circ}\right.$, and $8^{\circ}$ ); (c) depth (present or absent); and (d) shape (fat-vertical segments bowed out, horizontal segments bowed in - and thinvertical segments bowed in, horizontal segments bowed out). This $4 \times 4 \times 2 \times 2$ within-subjects design yielded 64 different stimulus displays. These 64 trials formed a block of trials. Data were collected from six blocks per participant.

Stimuli and apparatus. The presentation of stimuli and the collection of participants' responses were controlled by a program written using MacProbe (Hunt, 1994), run on an Apple Macintosh IIfx computer. Displays were presented on an E-Machines TX16 monitor with a resolution of $87 \mathrm{dpi}(1,024 \times 808$ pixels). Responses were entered onto a Macintosh extended keyboard. Using a chin rest to stabilize their heads at a distance of $1 \mathrm{~m}$ from the monitor, participants viewed the displays through a stereoscope (Bernell Corporation, South Bend, IN). The stereoscope contained 8.5-diopter wedge prisms, base temporal. Given the screen separation of the views of the two eyes $(17 \mathrm{~cm})$, this produced a virtual distance of optical infinity. Spherical lenses of 1 diopter were used to match accommodative to convergence distance.

Each stimulus display consisted of a stereo pair, viewed through a stereoscope attachment to a cathode-ray tube screen. Each eye's view contained four black inducing elements on a white background (see Figure 5). The elements inducing an illusory (modal) 
figure were black three-quarter disks. The elements inducing an occluded (amodal) figure were the black three-quarter disks with a black line ( 5.82 min thick) completing the perimeter of the circle. The elements inducing the quasimodal display were both modal and amodal inducing elements, with two of each type placed along opposite diagonals. All inducing elements subtended $1.6^{\circ}$ of visual angle, with the entire display of four elements subtending $4^{\circ}$. The ratio of the visible edge length to the entire edge length (support ratio) was 0.65 .

Half of the displays incorporated depth information congruent with the type of figure induced: In the illusory (modal) display, the central illusory figure was placed in front of the inducing elements with a binocular disparity of $3.88 \mathrm{~min}$. In the occluded display, the central occluded figure was placed behind the inducing elements by the same amount. The disparity between inducing elements and their central figure was preserved in the quasimodal display so that the illusory inducing elements were placed $3.88 \mathrm{~min}$ behind the central figure, and the occluded inducing elements were placed $3.88 \mathrm{~min}$ in front of the central figure. The remaining half of the displays did not contain stereoscopic disparity differences; the same image was presented to each eye.

The misaligned-element controls were created by taking a quasimodal figure and shifting the inducing elements in the upper left and the lower right toward the center of the induced figure $11.64 \mathrm{~min}$ vertically and $11.64 \mathrm{~min}$ horizontally.

For all display types, the inducing elements were rotated $2^{\circ}, 3^{\circ}$, $4^{\circ}$, and $8^{\circ}$ to form potential figures that were either thin-bowed in along the vertical axis and bowed out along the horizontal axis-or fat-bowed out along the vertical axis and bowed in along the horizontal axis.

Procedure. Participants were told to decide whether the shape they perceived was "thin, where the [vertical] sides were bowed in, or fat, where the [vertical] sides were bowed out." These instructions were accompanied with hand gestures sketching vertical sides bowing in and bowing out. Participants were also told that some shapes would be slightly misaligned and that they were to perform the task as best they could in those cases. Participants were asked to respond as quickly and as accurately as possible by pressing with their right index finger the 9 key on the keypad for fat responses and with their left index finger the $q$ key on the keyboard for thin responses. The two keys were relabeled $\mathrm{F}$ and $\mathrm{T}$ as appropriate.

Before starting the practice trials, participants were asked to view stereoscopically a display showing a square frame, subtending $4.64^{\circ}$, containing two black dots, with diameters subtending $38.81 \mathrm{~min}$. One dot was placed behind the depth of the frame, the other in front, with a horizontal disparity of $3.88 \mathrm{~min}$. Participants were asked if they could determine which of the two dots was closer to them. Participants who were unable to see stereoscopic depth were given credit and did not participate further in this experiment. Throughout all the experiments, less than $5 \%$ of the participant pool was excused at this point.

Participants then began a block containing all 64 trials as practice trials. Data from the practice block were not included in the statistical analyses. As an additional test of stereo vision, participants were asked if they could see the depth information in the practice trials before they continued further. Participants then ran an additional six blocks of trials. All trials were randomized within a block before each iteration. Participants received feedback about their performance by means of an auditory "incorrect" signal.

Each participant performed the experiment individually in a room with standard fluorescent illumination.

\section{Results}

Accuracy was high (averaging $98 \%$ correct) and did not differ reliably across groups. Reaction times (RTs; correct responses only) for each display type are shown in Figure 6 as a function of angle of rotation. Increasing rotation angle led to shorter RTs. The patterns for the illusory object, occluded object, and quasimodal displays appear virtually identical; the control group showed slower responding by about $200 \mathrm{~ms}$ (roughly $25 \%$ ) at all rotation angles.

These observations were confirmed by the analyses. A 2 (depth) $\times 4$ (inducer rotation) $\times 4$ (inducing element type) repeated-measures analysis of variance (ANOVA) found effects of inducer rotation, $F(3,42)=9.01, p<.001$, and inducing element type, $F(3,42)=11.34, p<.0001$. There was no main effect of depth $(F<1)$, nor were there any interactions involving this factor. In subsequent analyses, data were collapsed across depth. There was no reliable interaction of display type and rotation $(F<1)$.

Planned comparisons showed that the main effect of inducing element type was due to slower responding to the misaligned-element control displays, $F(1,14)=12.28, p<$ .005 , whereas pairwise comparisons of other conditions did not reach significance. A test for interacting contrasts found that this pattern of results did not differ with inducer rotation $(F<1)$.

The inducer rotation effect, tested with pairwise comparisons, found RTs to be significantly different between all conditions. Reaction times increased as inducer rotation increased.

\section{Discussion}

The results of Experiment 1 indicate that displays known to induce object completion produce a performance advantage relative to a control group in which completion was disrupted. Using a slightly different version of Ringach and Shapley's (1996) classification task and a different type of

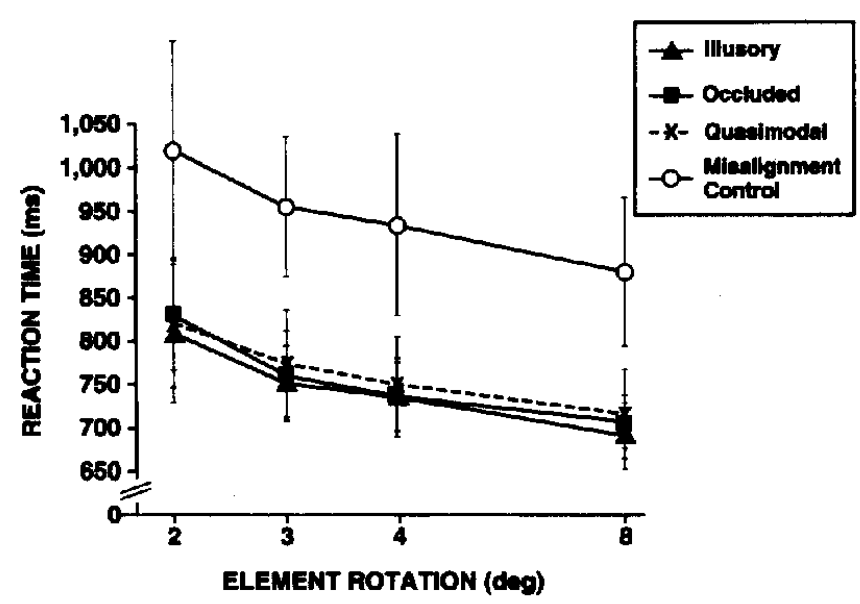

Figure 6. Reaction times (in milliseconds) in Experiment 1 for each display type, plotted as function of inducer-element rotation. Error bars show $\pm 1 S E$ of the mean. 
control display, we confirmed their findings. Our results also indicate no differences in the processing of illusory and occluded displays, providing further support for the identity hypothesis (Kellman \& Shipley, 1991).

Disruption of object completion by misalignment of parallel contours (control group) confirms the model of contour relatability put forth by Kellman and Shipley (1991). Although the present experiment tested only a single value of misalignment (about $11.64 \mathrm{~min}$ ), disruption of the object completion effect was substantial. The result provides evidence from performance data that fits well with earlier perceptual report results (Shipley \& Kellman, 1992).

The most important result of Experiment 1, however, is the finding that quasimodal displays showed the same patterns of performance as did illusory and occluded displays. These results confirm the phenomenological observation that quasimodal displays support object completion. The finding that illusory boundaries and surfaces can connect with occluded ones is a novel and stronger kind of evidence supporting a unitary completion process rather than separate processes for completing objects that appear behind or in front of other objects. Object completion occurs in displays that are neither amodal nor modal (or both amodal and modal).

\section{Experiment 2}

The findings of Experiment 1 were consistent with the hypotheses that (a) figural completion produces a performance advantage in the fat-thin discrimination task and (b) completion in displays having different appearances is the result of a common underlying mechanism. It remains possible, however, that performance differences found between completion and control displays in this study did not depend on figural completion. Perhaps misalignment of the elements made the processing of their rotations more difficult. Likewise, in Ringach and Shapley's (1996) displays, perhaps reversing element orientation caused distraction or otherwise reduced performance. An ideal control group would be one in which the boundaries relevant to the fat-thin detection task could be kept in identical positions in experimental and control groups. In Experiment 2 we sought to eliminate ambiguity about the cause of performance advantages in this task by testing control displays with contours in identical positions to those in the experimental displays.

To compare displays that should and should not show figural completion effects while keeping visible contours in the same location, we used illusory contour-type displays made of outline inducing elements. Illusory contours are weak or absent in displays made of outline inducers (Kanizsa, 1979; Kellman \& Shipley, 1991).

\section{Method}

Participants. Fifteen male and female undergraduates participated in partial fulfillment of requirements for an introductory psychology course at the University of California, Los Angeles. All participants had normal or corrected-to-normal vision, and none had participated in the previous experiment.
Design and stimuli. The same four independent variables from Experiment 1 were crossed. The only change in design was in the type of control displays. Instead of using misaligned-element controls, we used outline versions of the illusory object inducers. This $4 \times 4 \times 2 \times 2$ within-subjects design yielded 64 different stimulus displays. These 64 trials formed a block of trials.

Apparatus and procedure. The apparatus and procedure were the same as those in Experiment 1. Participants ran one block of 64 trials as practice and six blocks in the experiment.

\section{Results}

The main results are shown in Figure 7. Illusory object, occluded object, and quasimodal displays showed similar patterns of performance. Reaction times for the outline control displays were slower, on the order of $100 \mathrm{~ms}$, at each rotation angle. A 2 (depth) $\times 4$ (inducer rotation) $\times 4$ (inducing element type) repeated-measures ANOVA confirmed these observations. There were reliable main effects of inducing element type and inducer rotation, $F \mathrm{~s}(3,42)=$ 14.52 and 7.60 , respectively, $p s<.0001$. There were no main effects or interactions involving depth; data in subsequent analyses were collapsed across this variable. There was an interaction of inducing element type and inducer rotation, $F(9,126)=2.70, p<.01$.

Pairwise comparisons on the main effect of inducer rotation showed that RTs in the $2^{\circ}$ rotation condition were reliably $(\alpha=.05)$ slower than in the $3^{\circ}$ inducer condition. Responses times in the $3^{\circ}$ and $4^{\circ}$ conditions did not differ $(F<1)$. Responses times were reliably slower in the $4^{\circ}$ than in the $8^{\circ}$ condition.

The Inducer Type $\times$ Rotation interaction, which was not found in Experiment 1, was explored in two ways. The combined data from the completion groups (illusory, occluded, and quasimodal) were compared with the outline control group in a 2 (inducer type) $\times 4$ (rotation) ANOVA. The completion groups were faster than the outline control group, $F(1,14)=18.45, p<.001$, but the magnitude of this difference decreased with inducer rotation, $F(3,42)=3.1$,

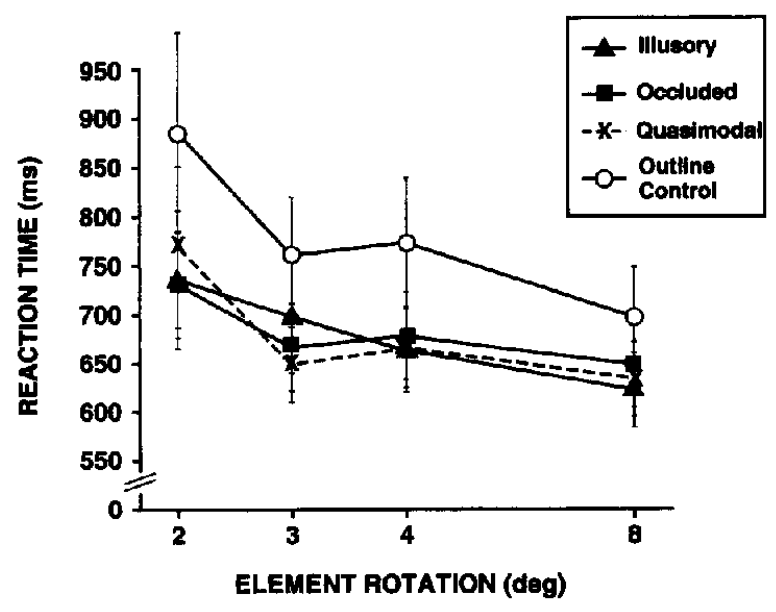

Figure 7. Reaction times (in milliseconds) in Experiment 2 for each display type, plotted as function of inducer-element rotation. Error bars show $\pm 1 S E$ of the mean. 
$p<.05$, as tested by the Inducer Type $\times$ Rotation interaction. Post hoc comparisons showed that this interaction was due to a greater difference between control and completion conditions when the inducers were rotated $2^{\circ}$ than when inducers were rotated $8^{\circ}, F(1,14)=7.02, p<$ .02 . The difference between control and test conditions for the $3^{\circ}$ and $8^{\circ}$ rotations was marginally significant, $F(1,14)=$ $3.47, .05<p<.10$.

To check for possible differences within the completion groups, a 3 (inducer type: illusory, occluded, quasimodal) $\times$ 4 (rotation) ANOVA was carried out with the control condition removed. There was no main effect of inducer type $(F<1)$, a main effect of inducer rotation, $F(3,42)=6.91$, $p<.001$, and a reliable interaction, $F(6,84)=2.36, p<$ .05 . Post hoc (simple effects) tests following up this $3 \times 4$ interaction found an interaction only between quasimodal and illusory conditions, $F(3,42)=3.13, p<.05$. Individual comparisons between quasimodal and illusory conditions at each rotation showed no reliable $(p<.05)$ differences, all $F \mathrm{~s}(1,14)<2.32, p s>.14$, and a marginally significant difference only at the $3^{\circ}$ rotation, $F(1,14)=3.43, p<.10$. Individual comparisons between all possible pairs of completion conditions at all rotations showed no other reliable differences.

A final analysis compared performance in the control conditions of Experiments 1 and 2. A 2 (experiment) $\times 4$ (rotation) ANOVA showed no main effect of experiment, $F(1,28)=2.10(n s)$, a reliable main effect of inducer rotation, $F(3,84)=7.19, p<.0002$, and no interaction $(F<1)$.

\section{Discussion}

The results of Experiment 2 generally mirror those of Experiment 1. Quasimodal, illusory, and occluded displays were all processed similarly, whereas the outline control displays were processed more slowly. An exception was the marginal superiority of quasimodal over illusory displays at the $3^{\circ}$ rotation. Because this effect is isolated, weak, and did not appear with the identical displays in Experiment 1, we are inclined to consider it a statistical fluke.

This experiment bolsters the interpretation that object completion produces the performance differences in this paradigm. Comparison of completion displays with outline displays fulfills the important criterion of preserving the locations of the contours providing local information for the discrimination task. The present results also confirm earlier claims that outline inducing elements do not produce illusory contours and figures (Kanizsa, 1979; Kellman \& Shipley, 1991). To our knowledge, the present result is the first experimental evidence for this claim derived from speeded performance rather than from perceptual report measures.

The lack of any difference in performance in the two control conditions used in Experiments 1 and 2 suggests that participants may have used a similar process to detect element rotation in the two cases.

\section{Experiment 3}

There is a final reservation to consider, however. It is possible that contour processing in outline displays is slower than in filled displays. If so, this difference could have produced the performance difference in Experiment 2. If there is no true object completion advantage in this task, then, conspiratorial as it might seem, the different control displays in Experiments 1 and 2 (and in Ringach \& Shapley's, 1996, experiments) might have each slowed performance for separate reasons. Evidence against the slower-or different-processing of outline displays would more convincingly establish the source of the performance advantage as object completion. To this end, we carried out Experiment 3.

In this experiment, a single illusory contour inducing element was used in the classification task. This element was an outline element on half the trials and a filled element on the other half. If contours in outline displays are processed more slowly than in filled ones, then we would expect to find faster classification of filled displays. If, however, the effect in Experiment 2 was truly due to object completion, we would expect to find no difference in speed of classifying single elements.

A second motivation for Experiment 3 was to test one idea about processing in the control groups of Experiments 1 and 2 . In the absence of figural completion, participants may perform the classification task by detecting orientation information in a single element. If performance with a single outline element shows the same pattern as that of the outline control group in Experiment 2, the outcome would support the idea that the task was performed in the control group without using interelement relationships (in contrast to the completion groups).

\section{Method}

Participants. Fifteen male and female undergraduates participated in partial fulfillment of requirements for an introductory psychology course at the University of California, Los Angeles. All participants had normal or corrected vision, and none had participated in the previous experiments.

Design. The variables of element type (filled or outline), shape (fat or thin), and inducer rotation $\left(2^{\circ}, 3^{\circ}, 4^{\circ}\right.$, and $8^{\circ}$ ) were fully crossed in a within-subjects design. This yielded 16 trials, which composed a block of trials. Participants ran 1 block of 16 trials for practice, and then 10 additional blocks for the experiment.

Stimuli and apparatus. The stimuli were created by using only the inducing element in the bottom left of the illusory and outline displays from Experiment 2. No disparity information was presented. Each display consisted of the inducing element presented in the center of the screen. Stimuli were presented on a Mitsubishi 20 in. $(0.51 \mathrm{~m})$ DiamondVision monitor, controlled by a MacProbe program running on a PowerMacintosh 7100 .

Procedure. Participants were seated $1 \mathrm{~m}$ from the monitor and, as in Experiments 1 and 2, were told they were to decide whether the shape they perceived was fat or thin. Examples of fat and thin figures were shown. Participants were then told that this shape judgment could be made using only part of the display, namely the bottom-left portion of the display. The experimenter then covered all of the display except the inducing element in the lower left and 
indicated the correct response of "fat" when the single element was tilted to the left and "thin" when it was tilted to the right. Other details of the procedure were identical to those of the previous experiments.

\section{Results}

The main results are shown in Figure 8. There was no difference in performance for outline and filled singleelement displays. A 4 (inducer rotation) $\times 2$ (inducing element type: outline or filled) repeated-measures ANOVA showed a reliable main effect of inducer rotation, $F(3,42)=$ $33.79, p<.001$, but no effect of inducing element type $(F<1)$. There was no reliable interaction.

Pairwise comparisons showed that the inducer rotation effect was similar to that found in Experiment 2: Reaction times generally decreased as inducer rotation increased, with the exception that rotations of $3^{\circ}$ and $4^{\circ}$ showed no RT difference $(F<1)$.

The single elements in the filled condition of this experiment were identical to elements used in Experiment 1 in the misaligned-element control condition. The single elements in the outline condition of this experiment were identical to elements used in the outline control condition of Experiment 2. To test the interpretation that in the noncompletion conditions of Experiments 1 and 2, participants used local orientation information from single elements, we compared performance on the single-element filled displays to the four-element, filled, misaligned displays of Experiment 1, and we compared the single-element outline displays with the four-element, outline displays used in the control condition of Experiment 2 . For the single versus multiplemisaligned elements, a 2 (experiment) $\times 4$ (rotation) ANOVA showed a main effect of rotation, $F(3,84)=9.70, p$ $<.0001$, no reliable main effect of experiment, $F(1,28)=$ $2.35(n s)$, and no interaction $(F<1)$. For the single versus multiple-outline element displays, a 2 (experiment) $\times 4$ (rotation) ANOVA showed a main effect of rotation, $F(3,84)$ $=27.55, p<.0001$, no reliable main effect of experiment $(F<1)$, and no interaction $(F<1)$.

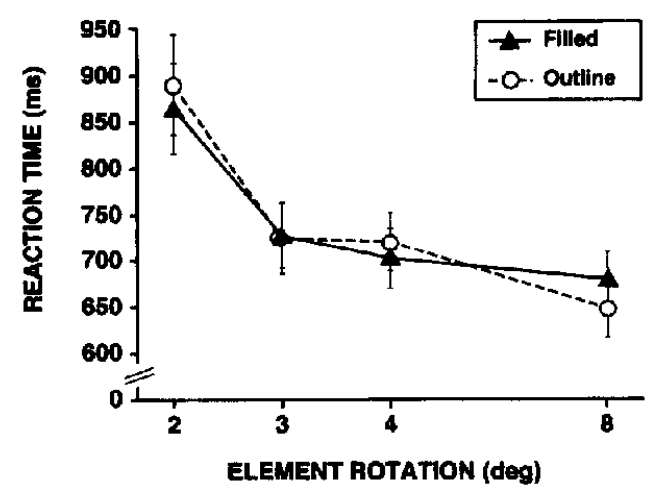

Figure 8. Reaction times (in milliseconds) in Experiment 3 for filled and outline single-element displays. Error bars show $\pm 1 S E$ of the mean.

\section{Discussion}

The results of Experiment 3 clarify the interpretation of Experiment 2. Individual outline elements were processed similarly to filled ones. Therefore, the effect of the outline control displays in Experiment 2 was not likely caused by some generally slower processing of outline edges relative to edges of filled areas. Participants' slower performance on outline displays in Experiment 2 indicates an object completion advantage for quasimodal, illusory, and occluded displays. The object completion process that produces this advantage is not engaged by outlined elements.

Comparisons of processing between Experiment 3 and earlier control conditions showed no reliable differences, suggesting that in the noncompletion conditions, participants' responses are based on local element orientation information. This must have been the case in Experiment 3, in which only a single element was presented on each trial. The close correspondence of response patterns between the single outline element in Experiment 3 and the outline displays in Experiment 2 suggests that participants did not use any interelement relationships in performing the task in control conditions, in contrast to the completion conditions.

\section{General Discussion}

These experiments support several general conclusions. First, the data strengthen the evidence that the fat-thin classification task detects processing advantages produced by object completion (Ringach \& Shapley, 1996). Previous research showed this advantage primarily with respect to one kind of control display: Two inducing elements were mirror-reversed to disrupt completion (Ringach \& Shapley, 1996). In another experiment, additional lines were interposed to interfere with completion. Almost any control condition taken in isolation is susceptible to alternative interpretations. For example, the mirror-image manipulation changes the positions of some display contours, which may have unknown effects on processing. One particular change is that it orients the concavities in two elements outward, disrupting completion but also making it harder for a viewer to imagine a figure enclosed by the elements. In the misaligned-element control condition of Experiment 1, all concavities remained inward toward a central region. In the outline control condition of Experiment 2, element contours matched the locations in the completion displays. A possible alternative explanation, based on speed of processing, for performance with outline controls was ruled out by Experiment 3.

These additional controls implicate object completion as the cause of the performance advantage. In every condition of every experiment, the correct response could be determined from the orientation of two perpendicular edges in any single element. Performance across conditions, however, showed a clear pattern of variation. Illusory, occluded, and quasimodal object displays were processed faster than displays that should have disrupted object completion.

Second, the identity hypothesis is supported in two ways by these experiments. Illusory and occluded object displays 
were always processed similarly, replicating earlier results suggesting common underlying mechanisms. What is more, tests of a new class of displays showed that illusory contours and occluded contours can join. The joining occurs under conditions that fulfill neither the requirements for illusory contour completion alone nor occluded contour completion alone. Thus, the completed boundaries and figures are neither strictly modal nor amodal. They are both, or neither, or perhaps quasimodal. The existence of quasimodal displays argues against the notion that edge formation can be separated into separate processes of modal and amodal completion. The fact that quasimodal displays are processed similarly to pure illusory and occluded displays provides objective evidence for completion in these new displays and is consistent with their dependence on the same mechanisms that produce occluded and illusory edges.

Other phenomena provide related logical arguments that edge completion occurs in situations that cannot be classified as modal or amodal. Consider the display shown in Figure 9. (This stereopair should be free-fused or viewed in a stereoscope. The discussion below assumes free-fusing by crossing the eyes; all of the effects should occur for uncrossed viewing, but the depths are reversed.) A horizontal white bar is visible, slanted in depth around a vertical axis. It can be seen that the left end of the bar is nearest, the right end farthest, and the depth positions along the bar change smoothly from left to right. Most interesting is the appearance of visual interpolation along the bar. It has illusory contours (modal completion) between the left circular element and the middle of the center circular element. It then passes behind the right edge of the center element and becomes occluded (amodal completion) by the surrounding surface before appearing through a circular window on the right.

This display is constructed so that no depth information is given for the bar except at its ends. Crossed disparity places the left end closest to the viewer and the right end farthest. How do the remaining parts of the bar take on positions in depth? Part of the answer is not mysterious: Within an object, depth can "spread" between endpoints (Gillam, Chambers, \& Russo, 1988; Nakayama, Shimojo, \& Silver-

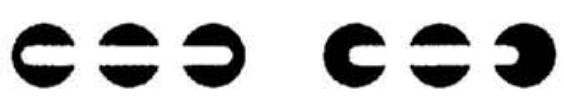

(a)

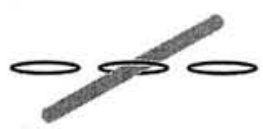

(b)

Figure 9. (a) Display in which contour interpolation must precede determination of modal or amodal appearance. Free-fuse by crossing the eyes. (b) Top view of the perceived 3-D arrangement in (a).

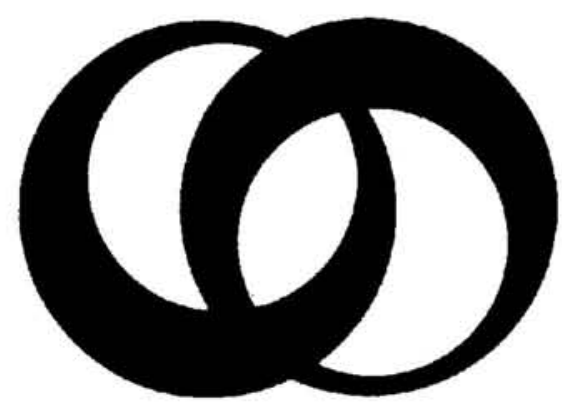

Figure 10. A spontaneously splitting object that illustrates the Petter (1965) effect. Despite homogeneous surface quality, the display is seen as containing two separate objects. The Petter effect refers to the following: Where regions of the two objects cross, the one having shorter interpolated boundaries appears in front. (Redrawn from Kanizsa, 1979.)

man, 1989). ${ }^{2}$ The instructive part of this example is the interaction between object completion and depth positioning. Depth does not spread to all points of the white surface: It spreads within the object's interpolated contours. This means that object completion must occur before the intermediate positions assume their perceived depth values. Yet the appearance of the completed contours and surfaces as amodal (occluded) or modal (illusory) depends on these depth values. Therefore, it makes no sense to attribute part of the boundary interpolation here to an amodal completion process and part to a modal completion process. The interpolation process logically precedes the determination of modal or amodal character.

Similar considerations apply to interpolation of the boundaries of the middle circular element. It has an occluded contour boundary on the left and an illusory contour boundary on the right. These apparent positions depend on the depth positions of parts of the interpolated horizontal bar. ${ }^{3}$

Although the example we have just discussed is new, there is a well-known phenomenon that suggests the same explanation. Figure 10 shows a type of display discovered by Petter (1965) and discussed by Kanizsa (1979). It involves SSOs, in which homogeneous regions appear to split into two segregated objects. Petter noticed that when the interpolated boundaries of the two objects cross, there is a rule that governs which appears in front and which behind. The interpolated boundary that traverses the smaller gap tends to appear modally (in front), whereas the boundary that traverses the larger gap appears amodally (behind). The

\footnotetext{
${ }^{2}$ We avoid the commonly used term disparity spreading because it is perceived depth that spreads, not the stimulus information given by retinal disparities.

${ }^{3}$ With prolonged viewing, a different appearance may be noted. The bar may appear to attach to the edge of the middle circular element. This appearance may reflect some depth-spreading competition from the middle element, and it appears to depend on where the observer fixates. We focus here on the immediate appearance of the display. We thank Barton Anderson for pointing out the alternative appearance as well as for useful discussions of depth spreading.
} 
logical implication is similar to that in the example above. The computation that determines depth ordering here compares the length of interpolated boundaries. It would appear, therefore, that interpolated boundaries must be registered before their appearance as modal or amodal can be determined.

It is important to avert a possible misunderstanding. These examples in which depth ordering logically must occur after boundary interpolation are rare. It does not appear that depth ordering is in general determined after boundary interpolation. It can be when displays (or display parts) have poorly specified depth to begin with or when the depth of certain surfaces is roughly the same. In many ordinary viewing situations, depth information about the positions of parts of objects is unambiguous. Such depth information provides an input to the unit formation process (Kellman \& Shipley, 1992), and indeed, differences in depth may prevent otherwise relatable object parts from joining perceptually (Kellman, Machado, Shipley, \& Li, 1996; Kellman \& Shipley, 1991). Also, the 3-D positions of other contours and surfaces may influence the strength of completion (Anderson \& Julesz, 1995; Nakayama et al., 1989). Although this point has perhaps been underemphasized, in our model of boundary interpolation (Kellman \& Shipley, 1991, 1992) depth is involved both at the beginning and at the end of the process. On the input side, depth relations are an important component of edge relatability, which determines edge interpolation. After interpolation occurs, final depth adjustments may occur as a result of new (interpolated) boundaries and surface regions (Kellman \& Shipley, 1991, 1992).

The logical impact of the new type of display (Figure 9) and Petter-effect displays, then, is not that interpolation must always precede depth positioning but that it does sometimes. The fact that completion mechanisms can operate on objects whose final depth order will be determined later, as a consequence of completion, precludes the notion that completion itself is modal or amodal. Likewise, the existence of quasimodal displays, which engage completion mechanisms similarly to occluded and illusory object displays, makes the same point in a different way. This conclusion rests on interpreting the terms amodal and modal (Michotte et al., 1964) as referring to the appearance, specifically the depth order, of perceived edges and surfaces. A modally completed surface is the nearest to the observer in its visual direction, whereas an amodally completed one is not-some other surface is in front. The logical point we are making is that this final appearance cannot be used to individuate two processes of completion. It is of course possible that there are multiple completion processes in the visual system and that they involve various neural mechanisms. But individuating these processes and mechanisms has to be done on some bases other than their ultimate modal or amodal appearance.

The findings and analyses indicating that edge completion can precede the determination of modal or amodal appearance in the final percept supports a common component in the generation of illusory and occluded objects. Not all aspects of these completion phenomena may be common, however. For example, the time required for initial registration of the visible parts of displays may differ (Ringach \& Shapley, 1996, Experiment 6). There is also some contro- versy as to whether illusory contour displays and occlusion displays have similar effects in visual search. Davis and Driver (1994) reported that illusory figure displays can be detected without focal attention (RT was roughly constant across distractor-set size), whereas displays with an additional line drawn in, much like the occlusion displays used in our experiments, required focal attention (RT increased with distractor-set size). Earlier, Grabowecky and Treisman (1989) reported that illusory contour displays also required serial search. The Davis and Driver paradigm was unique in that all elements first appeared as solid circles and then transformed. It is possible that "pop-out" in the illusory contour displays could have been mediated by information other than the presence of an illusory form. Perhaps participants were detecting the presence of smooth curves along the exterior of the display. Because the distractors were inducing elements that faced outwards, such regions only occurred when the illusory figure was present. This basis for discrimination was unavailable in the control conditions in which all displays were completely bounded by circles. Unfortunately, these issues make it hard to tell whether there is truly any difference between illusory and occluded figures in visual search. In any case, a difference in visual search could reflect differences in the way attention handles the outputs of object formation rather than differences in the formation processes themselves. Specifically, surfaces that end up being the nearest in their visual direction (modal) may be more likely to attract attention than those that end up in a further depth layer.

At the physiological level, results by Peterhans and von der Heydt (1991) suggest that certain V2 cells are stimulated when their receptive fields fall within the gaps of illusory contour displays, but this does not occur for a display with additional lines drawn in. This interesting result may suggest that although the interpolation process is similar across different displays, the actual neural mechanisms differ. It is difficult, however, to interpret with much confidence the function of single neural responses. Although these V2 cells could be computing illusory contours, they could be doing numerous other things. Also, controversy persists about the requirements that must be met to claim that a stimulus falls outside of a cell's receptive field (Bock \& Goode, 1994).

If there are differences in neural coding, our results pose some interesting questions: How are quasimodal displays encoded? What neural mechanisms operate in displays in which the occluded or illusory status of an edge is determined after interpolation of the edge? These questions will be important in guiding further research on what different completion phenomena do and do not have in common. Our results suggest, on empirical and logical grounds, that despite their differing phenomenology, illusory and occluded (modal and amodal) object completion depend on a common process of edge interpolation.

\section{References}

Anderson, B. L., \& Julesz, B. (1995). A theoretical analysis of illusory contour formation in stereopsis. Psychological Review, 102, 705-743.

Bock, G. R., \& Goode, J. A. (1994). Higher-order processing in the visual system. Chichester, England: Wiley. 
Brigner, W. C., \& Gallagher, M. B. (1974). Subjective contour: Apparent depth or simultaneous brightness contrast? Penceptual and Motor Skills, 38, 1047-1053.

Davis, G., \& Driver, J. (1994). Parallel detection of Kanizsa subjective figures in the human visual system. Nature, 371, 791-793.

Gillam, B., Chambers, D., \& Russo, T. (1988). Postfusional latency in stereoscopic slant perception and the primitives of stereopsis. Journal of Experimental Psychology: Human Perception and Performance, 14, 163-175.

Grabowecky, M., \& Treisman, A. (1989). Attention and fixation in subjective contour perception. Investigative Ophthalmology \& Visual Science Abstracts, 30(3), 457.

Gregory, R. (1977). Vision with isoluminant color contrast: 1. A projection technique and observation. Perception, 6, 113-119.

Grossberg, S., \& Mingolla, E. (1985). Neural dynamics of form perception: Boundary completion, illusory figures, and neon color spreading. Psychological Review, 92, 173-211.

Hunt, S. M. J. (1994). MacProbe: A Macintosh-based experimenter's workstation for the cognitive sciences. Behavior Research Methods, Instruments, \& Computers, 26, 345-351.

Jory, M., \& Day, R. (1979). The relationship between brightness contrast and illusory contours. Perception, 8, 3-9.

Kanizsa, G. (1979). Organization in vision. New York: Praeger.

Kellman, P., \& Loukides, M. (1987). An object perception approach to static and kinetic subjective contours. In S. Petry \& G. E. Meyer (Eds.), The perception of illusory contours (pp. 151-164). New York: Springer-Verlag.

Kellman, P. J., Machado, L., Shipley, T. F., \& Li, C. C. (1996). 3-D determinants of object completion. Investigative Ophthalmology \& Visual Science Abstracts, 37(3), 685.

Kellman, P. J., \& Shipley, T. F. (1991). A theory of visual interpolation in object perception. Cognitive Psychology, 23, 141-221.

Kellman, P. J., \& Shipley, T. F. (1992). Perceiving objects across gaps in space and time. Current Directions in Psychological Science, 1, 193-199.

Michotte, A., Thines, G., \& Crabbe, G. (1964). Les complements amodaux des structures perceptives [Amodal complements of perceptual structures]. In Studia psychologica. Louvain, Belgium: Publications Universitaires de Louvain.

Nakayama, K., \& Shimojo, S. (1990). Toward a neural understand- ing of visual surface representation. Cold Spring Harbor Symposia on Quantitative Biology, 55, 911-924.

Nakayama, K., \& Shimojo, S. (1992, September). Experiencing and perceiving visual surfaces. Science, 257, 1357-1363.

Nakayama, K., Shimojo, S., \& Silverman, G. (1989). Stereoscopic depth: Its relation to image segmentation, grouping, and the recognition of occluded objects. Perception, 18, 55-68.

Peterhans, E., \& von der Heydt, R. (1991). Subjective contours: Bridging the gap between psychophysics and physiology. Trends in Neurosciences, 14(3), 112-119.

Petter, G. (1965). Nuove ricerche sperimentali sulla totalizzazione percettiva. Rivista di Psicologia, 50, 213-227.

Ringach, D. L., \& Shapley, R. (1996). Spatial and temporal properties of illusory contours and amodal boundary completion. Vision Research, 36, 3037-3050.

Rock, I. (1987). A problem solving approach to illusory contours. In S. Petry \& G. E. Meyer (Eds.), The perception of illusory contours (pp. 62-70). New York: Springer-Verlag.

Shapley, R., \& Ringach, D. L. (1994). Similar mechanisms for illusory contours and amodal completion. Investigative Ophthalmology and Visual Science Abstracts, 35(4), 1491.

Shimojo, S., \& Nakayama, K. (1990). Amodal representation of occluded surfaces: Role of invisible stimuli in apparent motion correspondence. Perception, 19, 285-299.

Shipley, T. F., \& Kellman, P. J. (1992). Perception of partly occluded objects and illusory figures: Evidence for an identity hypothesis. Joumal of Experimental Psychology: Human Perception and Performance, 18, 106-120.

von der Heydt, R., Peterhans, E., \& Baumgartner, G. (1984, June). Illusory contours and cortical neuron responses. Science, 224 1260-1262.

Yarbus, A. L. (1967). Eye movements and vision. New York Plenum Press.

Yin, C., Kellman, P. J., \& Shipley, T. F. (1997). Surface completion complements boundary interpolation in the visual integration of partly occluded objects. Perception, 26, 1459-1479.

Received December 17, 1995 Revision received November 22, 1996 Accepted April 1, 1997 